\title{
Non-invasive assessment of aortic stenosis by Doppler ultrasound
}

\author{
L HATLE, B A ANGELSEN, A TROMSDAL \\ From the Section of Cardiology, Medical Department, University Hospital, Trondheim; and Division \\ of Engineering Cybernetics, The Norwegian Institute of Technology and Division of Automatic Control at \\ the Foundation of Scientific and Industrial Control at the University of Trondheim, Trondheim, \\ Norway
}

SUMMARY The peak pressure drop across the aortic valve in aortic stenosis has been measured by Doppler ultrasound. Maximum velocity in the Doppler signal from the aortic jet was recorded using a maximum frequency estimator. With an angle close to zero between ultrasound beam and maximal velocity in the jet, peak pressure drop can be calculated from the maximal velocity measured; a larger angle will underestimate maximal velocity and pressure drop.

In 57 of 63 patients with aortic stenosis, the aortic jet could be reached by the ultrasound beam and, in 37 of these, peak pressure drop by ultrasound was compared with that obtained at catheterisation.

In patients less than 50 years of age the aortic jet was easy to find, the measurement was reproducible, and underestimation of the pressure drop obtained at catheterisation was within 25 per cent in 17 of 18 patients. In patients over 50 years Doppler signals from the aortic jet were more difficult to obtain, and pressure drop was significantly underestimated in one-third, but time of maximum velocity in systole could indicate whether moderate or severe aortic stenosis was present.

In mitral stenosis it has been shown that maximal velocity in the mitral jet can be measured noninvasively by ultrasonic Doppler technique provided a small enough angle between the ultrasound beam and the mitral jet can be obtained. The pressure drop across the valve can be calculated from the maximal velocity, using the Bernoulli equation, and has been found to correlate well with results obtained simultaneously at catheterisation. ${ }^{12}$

In the present study the same method was used to see if the pressure drop across the valve in aortic stenosis could be obtained non-invasively by ultrasound. In 41 patients with aortic stenosis both ultrasound and catheterisation studies were done. In addition, 22 patients with aortic stenosis were studied by ultrasound alone. Among these 63 patients the aortic jet could be recorded by ultrasound in 57.

\section{Theoretical considerations}

In a previous study ${ }^{2}$ the Bernoulli equation was used to calculate the pressure drop in mitral stenosis from the increase in maximal velocity Received for publication 14 May 1979 across the valve, and the following formula was derived:

$$
P_{1}-P_{2}=4 \times V_{2}^{2}(\mathrm{mmHg})
$$

where $\mathrm{V}_{2}=$ maximal velocity measured in the jet $(\mathrm{m} / \mathrm{s})$. The velocity in the left atrium was assumed to be so low that it could be neglected.

An acceleration term originally included in the formula was considered to be of little importance except at valve opening and closure. Viscous losses were also considered, but comparing simultaneous velocity and pressure recordings in mitral stenosis indicated that these terms could be neglected at the velocities present in mitral stenosis.

In this study peak pressure drop across the valve in aortic stenosis was calculated from maximal velocity in the aortic jet measured by Doppler ultrasound using formula (1). The velocity in the left ventricular outflow tract, usually 0.7 to $1 \mathrm{~m} / \mathrm{s}$, was neglected. This can be recorded in most patients and becomes important in patients with additional subvalvular obstruction. The maximal velocity of the measured flow $\left(V_{2}\right)$ is obtained from the maximal frequency shift $\left(f_{2}\right)$ of the Doppler 
signal according to the following equation:

$$
V_{2}=\frac{C}{2 f_{0}} \cdot \frac{f_{2}}{\cos \theta}
$$

where $f_{0}$ is the transmitted ultrasonic frequency, $C$ the velocity of sound in blood, and $\theta$ the angle between ultrasound beam and the direction of maximal velocity.

To obtain the maximal velocity present in the measured area the angle between ultrasound beam and blood flow must be known or be so small that the cosine of the angle can be approximated to 1 and thus neglected in the equation. With an angle of $20^{\circ}$ between ultrasound beam and maximal velocity the velocity will be underestimated by 6 per cent. At a maximal velocity of $2 \mathrm{~m} / \mathrm{s}$ this will underestimate the pressure drop with 5 per cent and at $4 \mathrm{~m} / \mathrm{s}$ the underestimation of the pressure drop will be 11 per cent.

The direction of the aortic jet may vary considerably from patient to patient depending on the position of the heart and the aorta and on the aortic valve deformity. With the jet directed mainly upwards the smallest angle would most likely be found from the suprasternal notch or subxiphoid position. With a jet direction more to the right and anteriorly, measurements from the right sternal border-if possible-should give the smallest angle-while with a more posterior direction, aiming from the left sternal border or apex should give the best possibilities. The possible errors with this method therefore include:

Failure to reach the aortic jet.

Failure to obtain a small enough angle between ultrasound beam and maximal velocity.

Failure to maintain an adequate position throughout systole because of movements of the heart and the aorta. Errors introduced by neglecting viscous losses, shown to be unimportant at the velocities present in mitral stenosis.

In this study the ultrasonic and pressure measurements were not done simultaneously except in one case-and changes in cardiac output and peripheral resistance may have resulted in a different peak pressure drop on the two occasions.

\section{Patients and methods}

Mean age of the 63 patients was 46 (3 to 89) years. The age of the 41 patients with catheterisation studies varied from 10 to 71 years. Fifteen were less than 20 years, 14 were between 20 and 49 years, 27 patients were between 50 and 69 years, and seven were 70 years or more. Among patients below 20 years 11 were male and four were female, and among those above 20 years 28 were men and 20 women. In 33 patients aortic stenosis was the main valve lesion, 16 had moderate (11) to severe (five) aortic regurgitation, 10 had mitral stenosis, and five moderate to severe mitral regurgitation which, in one, was caused by a ruptured papillary muscle. In one of the 63 patients aortic regurgitation was combined with mitral stenosis.

The pressure drop across the aortic valve was measured by percutaneous retrograde catheterisation via the femoral artery in 35 patients, and by transseptal catheterisation in six. Pressures were recorded with an Elema transducer and an Elema Mingograph. The ultrasonic recording was done one day before catheterisation in most patients, and a few months later in two of the children; the blood pressure, however, was similar on the two occasions.

The apparatus used for the ultrasonic measurements is described in detail elsewhere. ${ }^{3-5}$ It can be used in either a pulsed mode when velocities up to $1.75 \mathrm{~m} / \mathrm{s}$ can be measured, or in a continuous mode when velocities up to $6 \mathrm{~m} / \mathrm{s}$ can be measured, but with loss of range resolution. To measure the velocities present in aortic stenosis the continuous mode must be used, while the pulsed mode is used to localise the increased velocities to the ascending aorta.

Several positions were tried to record aortic flow, the suprasternal notch, right and left sternal border, apex, and occasionally if these positions failed, the subxiphoid position. When aortic flow was obtained the transducer was moved/angulated to record flow just distal to the aortic valve (in the proximal part of the ascending aorta). In this position a harsh sound of high intensity caused by turbulence can be heard in aortic stenosis. This sound is clearly different from the sound of normal aortic flow. Exploring this area a more high-pitched sound may be recorded when the aortic jet is found. The highest velocity will be recorded in the area with the highest pitched sound. Mean and maximal velocity were then recorded together with the electrocardiogram and phonocardiogram on an Elema Mingograph with paper speed of 25, 50, and $100 \mathrm{~mm} / \mathrm{s}$. Peak pressure drop was calculated from the maximal velocity using formula (1).

The time from the start of flow to the peak of maximal velocity was also noted and divided by the duration of flow obtained from the flow curve recorded at $100 \mathrm{~mm} / \mathrm{s}$. Only beats where these points were clearly shown were used, and the mean of 5 to 10 beats was analysed. The resulting index ( $>0.50$ if peak velocity occurred after mid-systole) was compared with the pressure drop. This index 
was also measured in patients without heart disease and in patients with other valve lesions.

In some patients the ultrasonic examination was repeated on one or more occasions for up to two years.

The patients who were not investigated by methods other than ultrasonic examination were either small children or older patients where surgery was not indicated or was refused.

\section{Results}

Doppler signals from the aortic jet were easy to obtain in children and young patients, but at ages above 50 the success rate was much lower (Table 1 ). In six patients (aged 58 or more) aortic jet velocities could not be recorded, only a harsh sound indicating turbulence and the presence of aortic stenosis. Four of these had significant aortic stenosis and

Table 1 Doppler signals from the aortic jet obtained non-invasively

\begin{tabular}{lcll}
\hline & Good & Not satisfactory & Not obtained \\
\hline$<20$ years & 15 & - & - \\
$20-49$ years & 14 & - & - \\
$50-69$ years & 12 & 9 & - \\
$>70$ years & 2 & 5 & 6 \\
\hline & 43 & 14 &
\end{tabular}

two had moderate stenosis. Doppler signals from the jet were considered inadequate when only weak signals were obtained, or perhaps on only occasional beats or during part of systole. These 14 patients were over 58 years; 12 of them had significant aortic stenosis and two had moderate stenosis.

The position where the highest velocities were recorded in the 57 patients is given in Table 2 , as is the success rate of measuring from the right sternal border. Attempts at recording from the

Table 2 Positions for best ultrasonic recording of aortic jet velocity

\begin{tabular}{lll}
\hline & \multicolumn{2}{l}{ No. of patients } \\
\cline { 2 - 3 } & $<50$ years & $>50$ years \\
\hline Suprasternal notch & 16 & 13 \\
Right sternal border & 10 & 9 \\
Left sternal border & 1 & 2 \\
Apex & 1 & - \\
\hline Subxiphoid position & 1 & 28 \\
\hline & 29 & $33 \%$ \\
\hline $\begin{array}{l}\text { Aortic jet reached from right } \\
\text { sternal border }\end{array}$ & $70 \%$ & \\
\hline
\end{tabular}

right sternal border were not done in all the patients in the beginning of the study and only in the latter part were the patients turned over to the right side which usually made the recording of aortic jet velocities from this position easier. This may indicate that satisfactory recordings from the right sternal border can be obtained more often than is shown in Table 2.

Fig. 1 shows the maximal velocity curve recorded from the first right intercostal space in one of the patients. Maximal velocity recorded was $4.25 \mathrm{~m} / \mathrm{s}$ with a calculated peak pressure drop of $69 \mathrm{mmHg}$. At catheterisation a peak pressure drop of $68 \mathrm{mmHg}$ was found.

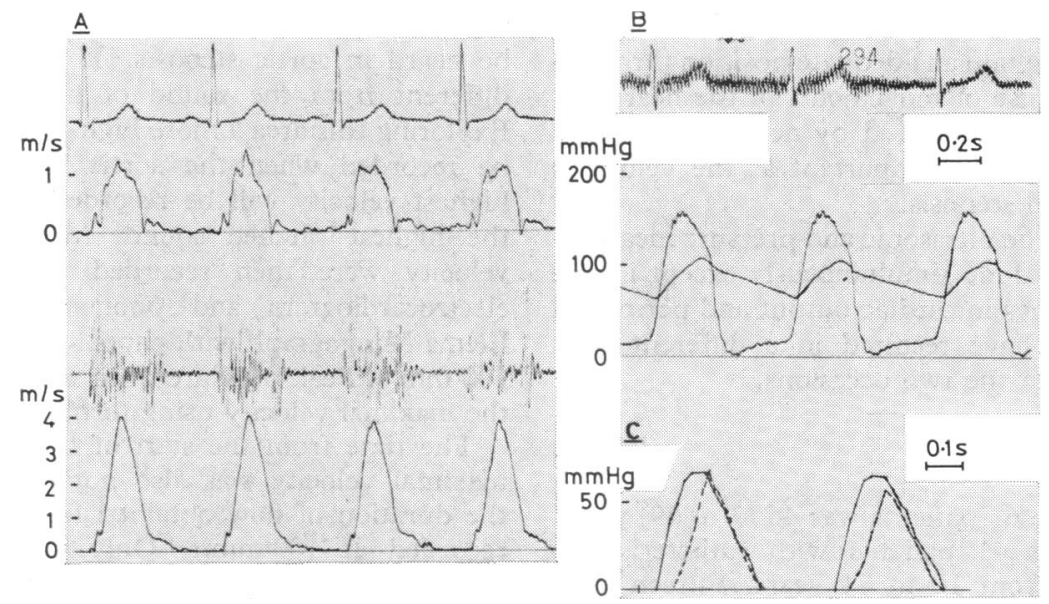

Fig. 1 (A) Ultrasonic recording of aortic flow from the first right intercostal space, mean and maximal (below) velocity; (B) pressure recording from the left ventricle and the aorta; (C) pressure drop across the aortic valve during systole for two beats. —— by pressure recording, ------ by ultrasonic recording. 


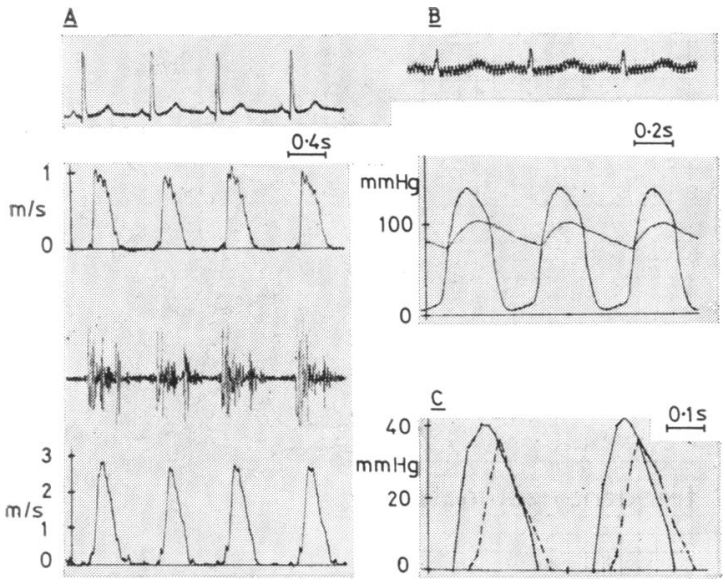

Fig. 2 (A) Mean (above) and maximal velocity of aortic flow measured by ultrasound from first right intercostal space; (B) left ventricular and aortic pressure; (C) Pressure drop across the aortic valve for two beats. -, pressure recording; -------, ultrasonic recording.

Fig. 2 shows velocity and pressure curves from another patient. Peak pressure drop at catheterisation was $42 \mathrm{mmHg}$, and maximal velocity recorded was $3.04 \mathrm{~m} / \mathrm{s}$ with a calculated pressure drop of $37 \mathrm{mmHg}$. Comparing the pressure drop during systole for the two methods (C), systole was slightly longer during ultrasonic examination when the heart rate was 80 than at catheterisation when the heart rate was 112 .

The delay seen in increase in velocity in the first part of systole compared with the pressure tracing was also found when velocity and pressure recordings were done simultaneously (Fig. 3). The maximal velocity curves for the two first beats are regular-giving no reason to suspect failure to record maximal velocities present in the first part of systole. The results in this case indicate that an angle close to zero between the ultrasound beam and maximal velocity was obtained in the first two beats.

The third beat shows an inferior signal but the timing of valve opening and closure is more clearly shown in the lowest curve where the amplitude of the Doppler signal is recorded.

In Fig. 4 the Doppler signal from the aortic jet is recorded simultaneously both with the maximum frequency estimator and a spectral analyser. Peak velocity is not well shown on spectral analysis, but it can be seen that velocities up to $4.5 \mathrm{~m} / \mathrm{s}$ are present. The maximum frequency estimator gives velocities of 4.90 to $4.97 \mathrm{~m} / \mathrm{s}$.

In Fig. 5 peak pressure drop at catheterisation is compared with that obtained by ultrasound in the 37 patients where the aortic jet was found. The pressure drop calculated from the ultrasonic recording was in almost all patients lower than that found at catheterisation.

In eight of the 37 patients the maximal velocity recorded resulted in a significant underestimation of the pressure drop. In seven of these clear signals from the aortic jet were difficult to obtain and made it unlikely that the maximal velocities present were recorded. In the eighth patient, however, flow signals from the jet were easily found and the recorded curves were regular, but maximal velocity and pressure drop were significantly underestimated. At repeat ultrasonic examination after catheterisation higher velocities were not found. In this case
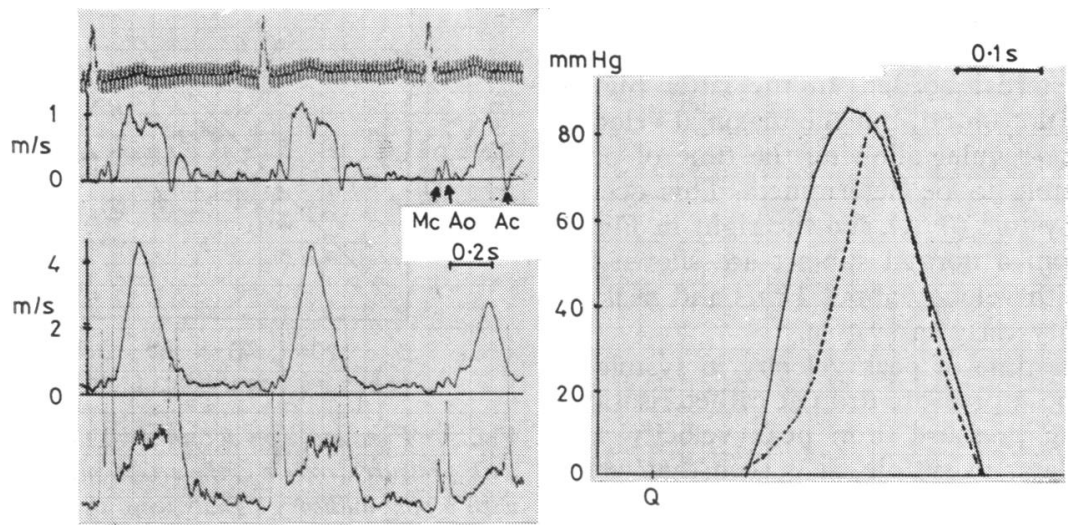

Fig. 3 Right: pressure drop recorded simultaneously at catheterisation. recording; $Q$, start of $Q R S ; M c$, mitral valve closure; Ao, aortic valve opening; Ac, aortic valve closure. Left: ultrasonic recording with mean and maximal velocity and amplitude of Doppler signal (lower curve where timing of valve motions is more clearly seen). 
Fig. 4 Simultaneous recording of Doppler signal from the aortic jet by maximal frequency estimator (A) and spectral analyser in one patient (B). (C) is from another patient where peak velocity in systole is better seen.
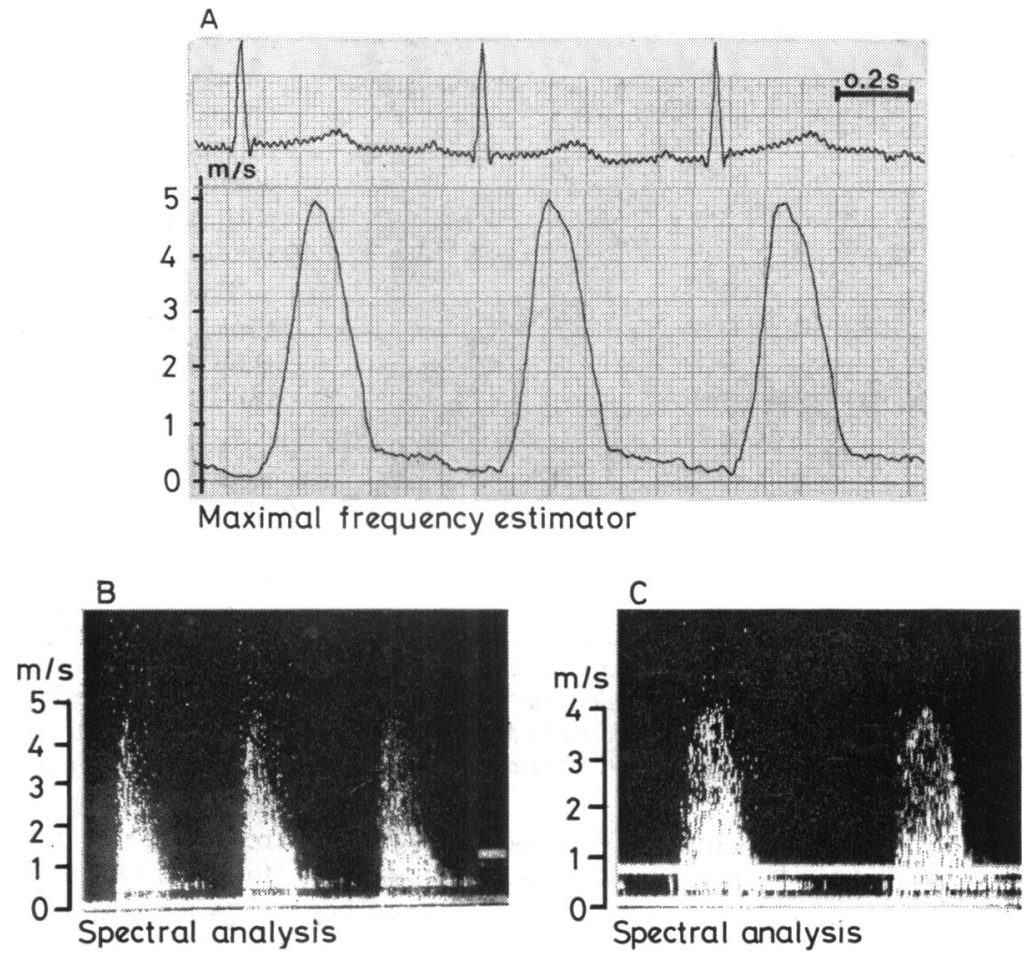

a small angle to the jet could probably not be obtained.

Fig. 6 is from a patient where Doppler signals from the aortic jet were difficult to obtain. The maximal velocity curve is irregular, indicating that jet velocities are only partly obtained. Maximal velocity recorded was $2.75 \mathrm{~m} / \mathrm{s}$ with a calculated pressure drop of $30 \mathrm{mmHg}$. At catheterisation, peak pressure drop across the valve was $130 \mathrm{mmHg}$. The recording to the left in Fig. 6 was made from the suprasternal notch, and the one in the middle from the left sternal border. In the latter higher velocities were not found, but the maximal velocity curve was more regular allowing the time of peak velocity in systole to be determined. This occurs well after midsystole (0.64). To the right in Fig. 6 flow curves from a normal subject are shown for comparison, with velocity about $1 \mathrm{~m} / \mathrm{s}$ and peak of maximal velocity early in systole.

In Fig. 7 the time of peak velocity in systole is related to the peak pressure drop at catheterisation. With increasing pressure drop peak velocity was usually found later in systole. It is seen that when peak velocity was later than midsystole $(>0.50)$ pressure drop was more than $50 \mathrm{mmHg}$ in almost all patients. The one exception with lower pressure drop and late peak velocity had severe mitral regurgitation with a short systole. 

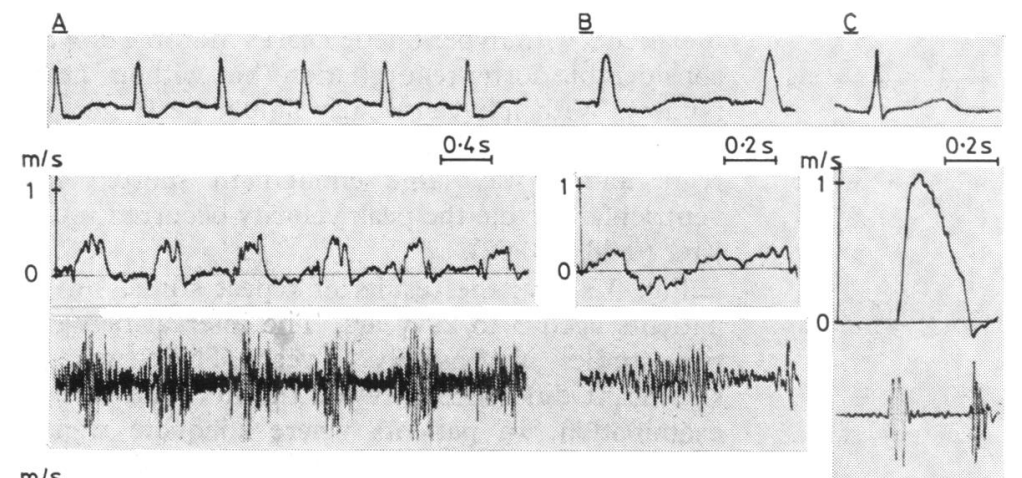

Fig. 6 Mean (above) and maximal velocity recorded from the suprasternal notch (A), from the fourth left intercostal space in the same patient (B), and in a normal subject $(\mathrm{C})$.
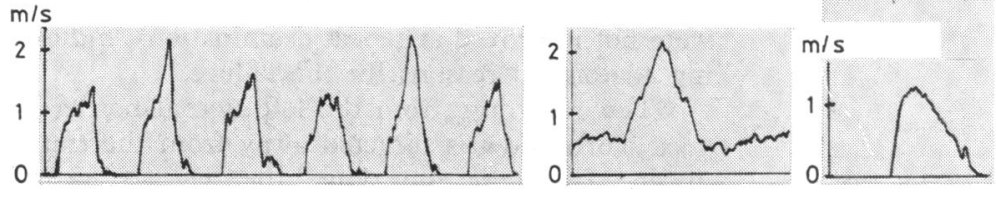

It is seen that peak velocity was late in systole in seven of the eight patients where maximal velocity was greatly underestimated. This may, therefore, indicate the presence of significant stenosis in these patients and that maximal velocity had not been obtained. The patient (marked with a square on the Figure) was the only one where peak velocity before midsystole was associated with a peak pressure drop greater than $50 \mathrm{mmHg}$; this patient also had massive aortic regurgitation.

The aortic jet was much easier to find in the young patient and the results were much better. The youngest patient whose pressure drop recorded by ultrasound was much lower than the actual drop was 45 years old (marked by a square in Fig. 5); the other patients where the maximal velocity

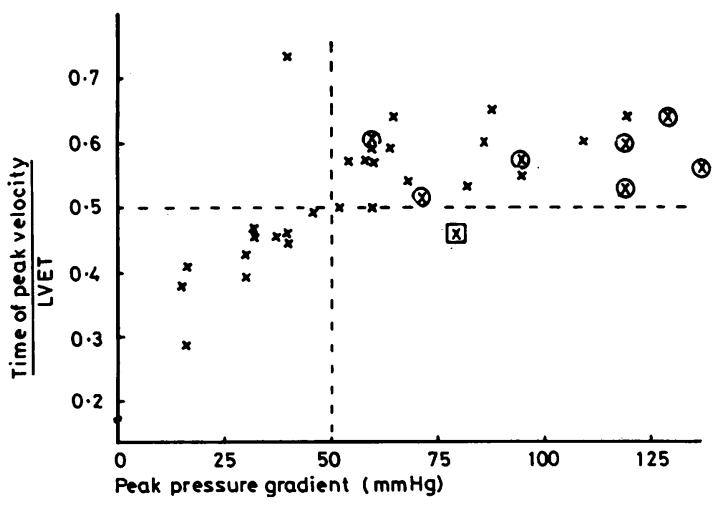

Fig. 7 Time of peak velocity in systole related to peak pressure gradient at catheterisation.

LVET, left ventricular ejection time.

The cases marked with rings or a square are the same as those in Fig. 5.

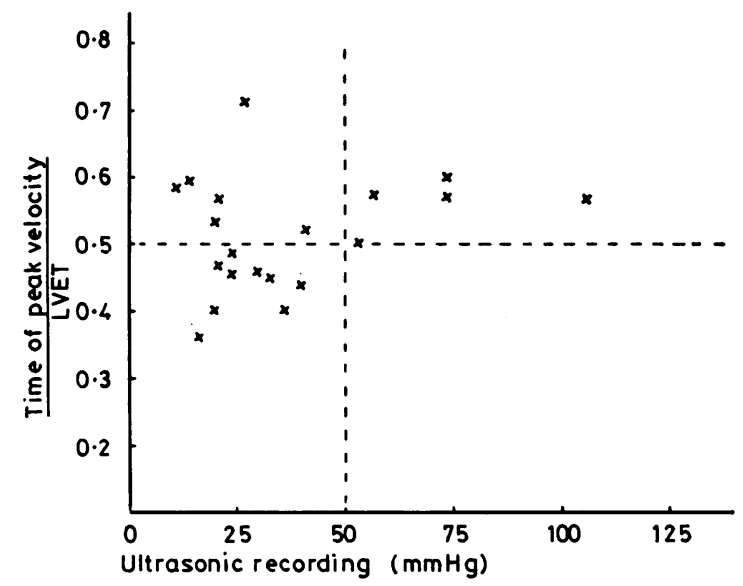

Fig. 8 Peak pressure drop from ultrasound in the patients without catheterisation related to time of peak velocity in systole.

recorded significantly underestimated the pressure drop were 58 to 70 years.

Fig. 8 shows the peak pressure drop obtained by ultrasound in 21 of the patients without a catheterisation study and related to the time of peak velocity in systole. The same relation as in Fig. 7 is found. In five of the patients with small pressure drop and late peak velocity, clear Doppler signals from the aortic jet were difficult to obtain and maximal velocity was probably much underestimated. In one of them (peak velocity at 0.71 ) the presence of severe aortic stenosis was confirmed at operation. The patients with latest peak velocity had additional severe mitral regurgitation.

In patients without heart diseease and in patients 


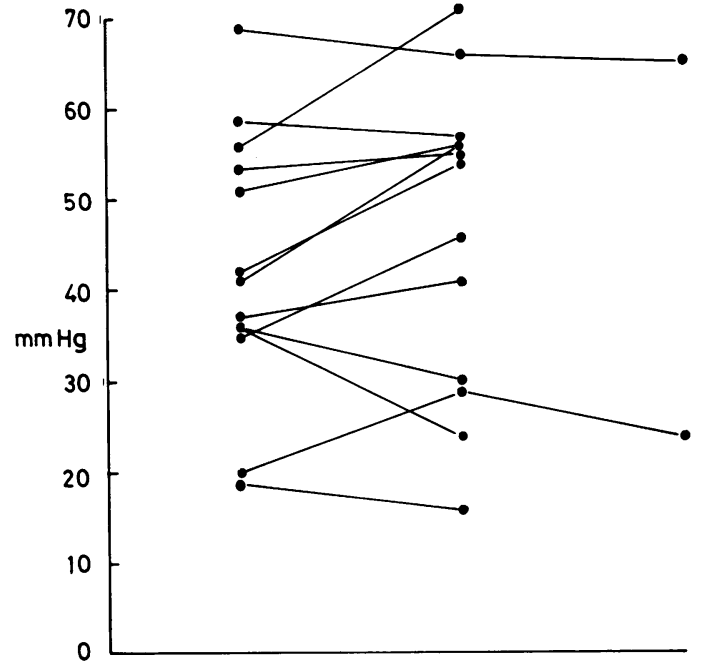

Fig. 9 Repeat examination in 13 patients. Time between first and second examination three to 15 months (mean 12.4).

with other heart diseases peak velocity in the ascending aorta occurred earlier except when left ventricular failure or severe mitral regurgitation was present.

In 40 children aged 1 to 20 years (mean 11) the time of peak maximal velocity was found at 0.30 (range 0.22 to 0.37 ) and was similar in 20 adults (range 0.21 to 0.35 ). The lowest values were seen in patients with hyperkinetic heart syndrome or with considerable aortic regurgitation (but without heart failure). With left ventricular failure peak velocity occurred later $(0.38$ to 0.41$)$ and when mitral regurgitation was large enough to shorten left ventricular systole the peak velocity occurred much later $(0.38$ to 0.52$)$.

Fig. 9 shows the results of repeat studies in 13 patients aged 3 to 29 years. The interval between two studies was usually a year. There was no significant difference between the first and second examination. In patients where adequate signals from the aortic jet could not be obtained, results were not improved at repeat examinations, indicating anatomical reasons for the failure.

When recording from the left sternal border or apex, aortic flow is directed away from the transducer. In these positions the jet of mitral regurgitation may be very close and in nearly the same direction. In mitral regurgitation even higher velocity may be present, especially in patients with high systolic pressure in the left ventricle where the pressure difference between the ventricle and atrium is large. In two of the patients with mitral regurgitation these high velocities were found in the blood flow back into the left atrium in addition to the high velocities in the aortic jet found in a slightly superior direction when recorded from the apex. Using pulsed ultrasound the high velocities could be located behind the mitral and aortic valves, respectively, in both cases. Flow in mitral regurgi-
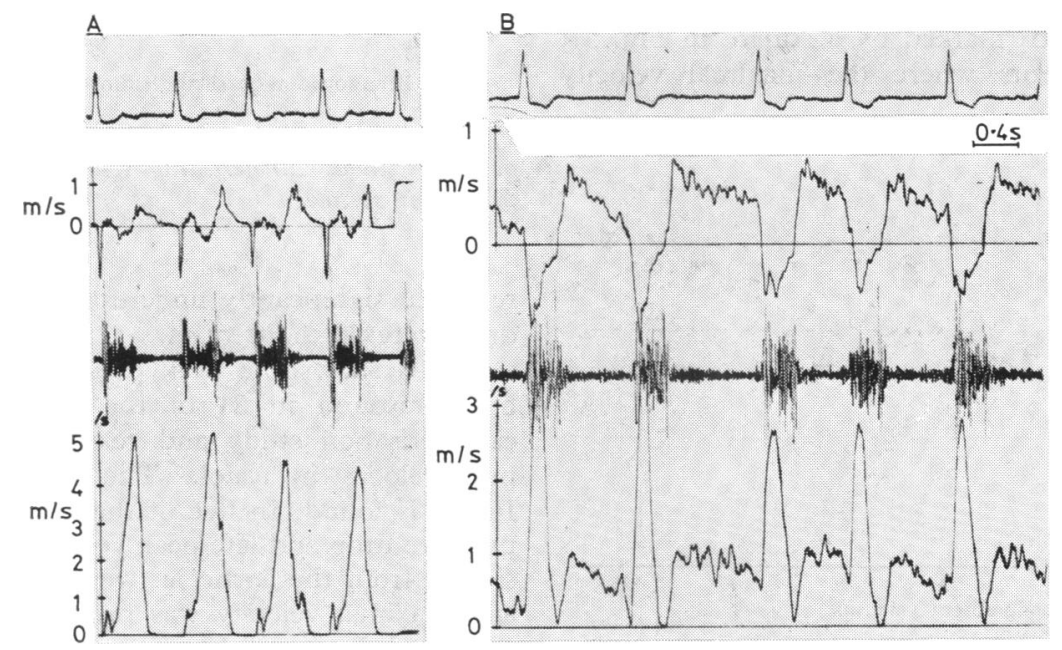

Fig. 10 Mean and maximal velocity curves recorded from the apex (with continuous ultrasound). Mean velocity is directive, flow towards the transducer is positive, and flow away from the transducer is negative. Maximal velocity is non-directive. (A) Aortic stenosis: mitral valve closure away from the transducer is also seen and in diastole some mitral flow; (B) mitral regurgitation. Flow is away from the transducer in systole with start of flow at mitral valve closure. In diastole flow into the left ventricle is recorded. 
tation also started earlier, at the closure of the mitral valve and maximal velocity was reached shortly after, compared with the later start of flow and later peak of maximal velocity in the aorta as shown in Fig. 10. Fig. 11 is redrawn from the angiogram showing the small angle between the aortic jet and mitral regurgitation in one of these. In the other cases of mitral regurgitation, flow also started at the closure of the mitral valve but lower and rather unchanged velocities during systole were found.

\section{Discussion}

The results in the patient with simultaneous ultrasonic and pressure recording, with a peak pressure drop of 84 and $86 \mathrm{mmHg}$, respectively, indicate that the applied formula can be used to calculate peak pressure drop from maximal velocity at the velocities present in aortic stenosis.

The delayed increase in velocity compared with the pressure recording is probably a result of the omission of a second acceleration term in the formula. As discussed in a previous paper, ${ }^{2}$ only convective acceleration is considered in the formula while acceleration resulting from changes in velocity with time was found to be of importance at the time of valve opening and during increase in velocity. At peak velocity this term could be ignored, and in mitral stenosis the period at the beginning of diastole where this term was of importance was so short that it could be ignored also in calculating mean pressure drop. That this delay is more evident in aortic stenosis may possibly be because

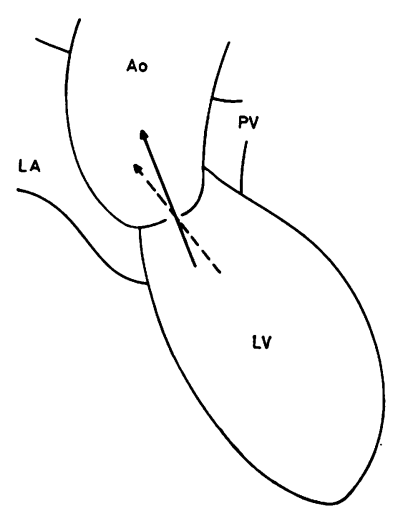

Fig. 11 Redrawn from the left ventricular angiogram from a patient with aortic stenosis and mitral regurgitation.

$L A$, left atrium; $L V$, left ventricle; $P V$, pulmonary vein; Ao, aorta. Solid arrows indicate aortic jet and stippled arrow indicates mitral jet.

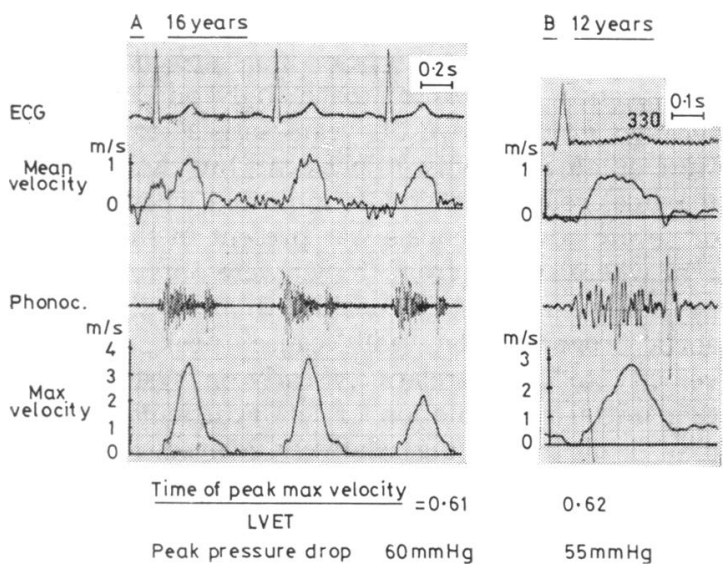

Fig. 12 Maximal velocity from the aortic jet shows peak velocity after mid-systole in both patients, and later in systole than peak murmur. Peak pressure drop at catheterisation is noted below.

of the longer acceleration period and the higher velocities. A possible delay caused by the frequency response of the maximal velocity estimator is calculated to be within five milliseconds and cannot fully explain the delay in the maximal velocity curve. Another possibility is that viscous losses may be of significance in aortic stenosis. This delay must be considered in the estimation of mean pressure drop in aortic stenosis but does not influence the calculation of peak pressure drop.

To obtain maximal velocity in the aortic jet noninvasively by ultrasound, the jet must be reached by the ultrasound beam and with a small angle between these to get a good estimate of the maximal velocity present in the jet. If the aortic jet is not reached no quantification of the pressure drop is possible, but a harsh sound of turbulence may still indicate the presence of aortic valve stenosis. Such a Doppler signal, instead of the more tone-like signal from normal aortic blood flow, has been reported as a reliable sign of aortic valve stenosis, ${ }^{6}$ and was present in all patients in this study, including those where signals from the aortic jet could not be obtained. If the aortic jet can be reached but with a substantial angle to the ultrasound beam maximal velocity and the pressure drop will be underestimated.

The difference in success rate with age both in finding the jet, obtaining good Doppler signals and good correlation to pressure may have anatomical reasons. With age, emphysema may limit the access to the ascending aorta, and increased valve deformity and calcification may produce greater variations in jet direction in the older patients. 
Below 50 years good Doppler signals were easily obtained in all, and peak pressure drop was significantly underestimated in only one of 18 . In the age group above 50 years satisfactory results were obtained in only 40 per cent, but time of peak maximal velocity in systole indicated whether mild or severe aortic stenosis was present in the 82 per cent where signals from the jet were obtained. The possibility must be considered that significant aortic regurgitation will cause peak maximal velocity to occur relatively early in systole; and that mitral regurgitation or left ventricular failure may result in it being relatively delayed.

In this study peak maximal velocity after midejection was almost always associated with a peak pressure drop above $50 \mathrm{mmHg}$. This may be compared with the timing of the maximal intensity of the systolic murmur, a late peak of murmur indicating a more severe stenosis. ${ }^{78}$ In children, the peak of the murmur may be unreliable with an early peak despite a high pressure drop. ${ }^{9}$ In the children in the present study peak velocity was found at or after mid-systole when peak pressure drop was above $50 \mathrm{mmHg}$, even if the peak of the murmur occurred earlier (Fig. 12).

In an effort to avoid underestimation of the pressure drop, several transducer positions should be tried and the region where the jet is found must be carefully scanned to obtain the best possible angle to the jet. When measurements are made from the apex or left sternal border care must be taken to distinguish between the jet of mitral regurgitation and the aortic jet. Subvalvular aortic stenosis can also be distinguished as the high velocity can then be localised to the left ventricular outflow tract below the aortic valve using pulsed ultrasound from the apical region.

Repeat measurements did not indicate any significant change in pressure drop over one to two years. This also indicates the reproducibility of the method, which may help in the non-invasive assessment of aortic stenosis provided the possibility of underestimating the pressure drop is kept in mind. It seems especially useful in children and young patients and in the follow-up of all patients where the jet is easily found.

This work was supported by the Norwegian Council on Cardiovascular Disease.

\section{References}

${ }^{1}$ Holen J, Aaslid R, Landmark $\mathrm{K}$, Simonsen S. Determination of pressure gradient in mitral stenosis with a noninvasive ultrasound Doppler technique. Acta Med Scand 1976; 199: 455-60.

${ }^{2}$ Hatle L, Brubakk A, Tromsdal A, Angelsen B. Noninvasive assessment of pressure drop in mitral stenosis by Doppler ultrasound. $\mathrm{Br}$ Heart $\mathcal{F} 1978$; 40: 131-40. ${ }^{3}$ Angelsen BAJ. Transcutaneous measurement of aortic blood velocity by ultrasound. A theoretical and experimental approach. Report 75-78-W. Norway: Division of Engineering Cybernetics, Norwegian Institute of Technology, University of Trondheim, 1975.

${ }^{4}$ Angelsen BAJ. Analog estimation of the maximum frequency of Doppler spectra in ultrasonic blood velocity measurements. Report 76-21-W. Norway: Division of Engineering Cybernetics, Norwegian Institute of Technology, University of Trondheim, 1976.

${ }^{5}$ Brubakk AO, Angelsen BAJ, Hatle L. Diagnosis of valvular heart disease using transcutaneous Doppler ultrasound. Cardiovasc Res 1977; 11: 461-9.

${ }^{6} \mathrm{Johnson}$ SL. Pulse-Doppler echocardiography. Localization of murmurs and spectrum of clinical applications. In: Mason DT, ed. Advances in heart disease, vol. 1. New York: Grune \& Stratton, 1977: 396.

'Oakley CM, Hallidie-Smith KA. Assessment of site and severity in congenital aortic stenosis. Br Heart $\mathcal{F}$ 1967; 29 : 367-79.

${ }^{8}$ Bonner AJ Jr, Sachs HN, Tavel ME. Assessing the severity of aortic stenosis by phonocardiography and external carotid pulse recordings. Circulation 1973; 48: 247-52.

${ }^{9} \mathrm{Gamboa}$ R, Hugenholtz PG, Nadas AS. Accuracy of the phonocardiogram in assessing severity of aortic and pulmonic stenosis. Circulation 1964; 30: 35-46.

Requests for reprints to Dr L Hatle, Section of Cardiology, University Hospital, 7000 Trondheim, Norway. 